\title{
New research perspectives on Ambient Intelligence
}

\author{
Emile Aarts ${ }^{\mathrm{a}, \mathrm{b}, *}$ and Boris de Ruyter ${ }^{\mathrm{a}}$ \\ ${ }^{a}$ Philips Research Europe, High Tech Campus 34, 6565 AA Eindhoven, The Netherlands \\ ${ }^{\mathrm{b}}$ Eindhoven University of Technology, P.O. Box 512, $5600 \mathrm{MB}$ Eindhoven
}

\begin{abstract}
Ten years of AmI research have led to many new insights and understandings about the way highly interactive environments should be designed to meet the requirement of being truly unobtrusive and supportive from an end-user perspective. Probably the most revealing finding is the fact that, in addition to cognitive intelligence and computing, also elements from social intelligence and design play a dominant role in the realization of the vision. In this paper we discuss these novel insights and their resulting impact on the AmI research landscape. We introduce a number of new AmI research perspectives that are related to social intelligence and in addition we argue that new ways of working are required applying the concept of Experience Research resulting in a true user-centered approach to Ambient Intelligence.
\end{abstract}

Keywords: Ambient Intelligence, natural interaction, user-centered design, experience research

\section{Introduction}

Developed in the late nineties of the past century, the Ambient Intelligence (AmI) paradigm presents a vision on digital systems for the years 2010 and beyond [3,5]. In an AmI world, massively distributed devices operate collectively while embedded in the environment using information and intelligence that is hidden in the interconnection network. Lighting, sound, vision, domestic appliances, personal healthcare devices, and distributed services all cooperate seamlessly with one another to improve the total user experience through the support of natural and intuitive user interfaces.

In short, Ambient Intelligence refers to electronic systems that are sensitive and responsive to the presence of people [2]. The foundation of the AmI vision is given by the fact that current technological developments will enable the integration of electronics into the environment, thus supporting the actors, i.e., people and objects to interact with their environment in a seamless, trustworthy, and natural manner. In addition, there is a growing desire that the role of information and communication technology should not be limited to productivity increase only. It also should support peoples' lives in terms of care, wellbeing, education, and creativity. In other words, novel technologies should not primarily increase functional complexity, but they should also contribute to the development of easy to use and simple to experience products and services, that make sense in the first place. Obviously, this desire is broadly endorsed by a wide range of scientists and engineers. However, reality reveals that it is hard to achieve in practice, and we need new insights to accomplish this formidable task. Nevertheless, during the past ten years substantial progress has been made and the literature presents many interesting achievements $[1,3,7]$.

\section{Related visions}

The AmI and many related visions build on the early ideas of Ubiquitous Computing as introduced in the 1990's by the late Marc Weiser [24] who anticipated a digital world in which electronic devices are the embedded parts of fine grained distributed net-

${ }^{*}$ Corresponding author. E-mail: emile.aarts@philips.com. 
Table 1

Positioning Ambient Intelligence

\begin{tabular}{lll}
\multicolumn{1}{c}{ Mobile } & \multicolumn{1}{c}{ Pervasive } & \multicolumn{1}{c}{ Ambient } \\
\hline - Portable & - Ubiquitous & - Embedded \\
- Wireless & - Interactive & - Context aware \\
- Networked & - Interoperable & - Personalized \\
- Location sensitive & - Distributed & - Adaptive \\
- Secure & - Scalable & - Anticipatory \\
\hline
\end{tabular}

works. Ubiquitous Computing provides a new view on mobile computing in the sense that mobile devices and their services become ubiquitously available providing access to secure data networks. The basic properties of mobile computing are well-known and can be summarized as follows [6]; see Table 1 .

- Portable. Small battery-operated handheld devices with large footprints and multi-functional properties.

- Wireless. Remote wireless connectivity with handover protocols and ad-hoc and TCP properties.

- Networked. Remote data and service access with layered protocols.

-Location sensitive. Global positioning with information on local position sensing.

- Secure. Encryption based with authentication and conditional access securing privacy.

Another interesting vision is that of Pervasive Computing, which stresses issues related to interoperability and seamless interconnectivity [20]. In a way, Pervasive Computing uses solutions from the mobile computing domain, but it also identifies some additional problems. The emphasis of Pervasive Computing is more on the software properties of services than on the device properties as in the case of mobile computing, which result from weight, size, and other physical constraints. The salient properties of Pervasive Computing can be formulated as follows:

- Ubiquitous. Overly present with identical appearance.

- Interactive. Control through multi-modal user interfaces.

- Interoperable. Plug and play with seamless integration and access.
- Distributed. Simultaneous access to resources including databases and processing units.

- Scalable. Adaptation of resources, quality of service and graceful degradation.

Ambient Intelligence aims at taking the embedding of devices one step further by involving the entire environment, i.e., any physical object, in the interaction with people, thus integrating electronics fully into the background of people with the purpose of improving productivity, creativity, and pleasure through enhanced user-system interaction. Evidently, the AmI vision uses solutions from the earlier visions on Mobile and Pervasive Computing. There are also new elements that call for novel approaches. These can be best explained from the two key words in the notion Ambient Intelligence.

The word ambience in Ambient Intelligence refers to the need for a large-scale embedding of technology in a way that it becomes non-obtrusively integrated into every-day objects and environments. The word intelligence reflects that the digital surroundings exhibit specific forms of social interaction, i.e., the environments should be able to recognize people, personalize to their individual preferences, adapt themselves to users over time, and possibly act upon their behalf. This implies that embedding through miniaturization is the main systems design objective from a hardware point of view. From a software point of view the main objective is to introduce true intelligence into these systems.

\section{AmIware}

Probably one of the most compelling developments in embedded technology or AmIware in brief is provided by Moore's law [17], which states that the integration density of systems on silicon doubles 
every eighteen months. This law seems to hold a selffulfilling prophecy because the computer industry follows this trend for already four decades. Moreover, other characteristic quantities of information processing systems, such as communication bandwidth, storage capacity, and cost per bit of input-output communication seem to follow similar rules. Moore's law rules the semiconductors industry for more than thirty years now and it provides a widely accepted forecast for the development of semiconductor technology. Recently, some new angles have been opened which in conjunction with Moore's law can be formulated as follows [3].

- 1D-Moore equals the one-dimensional continuation of the classical Moore's law into the submicron domain of micro-electronics, resulting in small and powerful integrated circuits that can be produced at low cost.

-2D-Moore is the development of 2-dimensional large-area electronic circuitry of extremely low cost, possibly using other technologies than silicon such as polymer-electronics.

$-3 \mathrm{D}$-Moore refers to the development of ultra-high functional 3-dimensional circuitry consisting of Micro Electronic Mechanical Systems (MEMS) or Systems in a Package (SIPs) that integrate sensor, actuator, computing, and communication functions into a singe nano-electronics system.

Recent technological developments in these three domains have opened the venue for new product breakthroughs [23]. The introduction of the blue laser in digital recording technology (DVR) has resulted in consumer devices that can record several tens of hours of video material, thus enabling time-shifted television watching. Solid-state storage technologies have resulted in portable music jukebox devices that can store thousands of songs. Poly-LED technology made it possible to construct the world's first matrix addressable display on a foil of a few micron of thickness, thus enabling the development of flexible ultra thin displays of arbitrary size. Developments in materials science have enabled the construction of electronic foils that exhibit paper-like properties. These so-called electronic paper devices introduce a new dimension in the use of electronic books or calendars. LCD projection allows very large highdefinition images being displayed on white walls from a small invisibly built-in unit. Advances in semiconductor process technology have made it possible to separate the active silicon area from its substrate, and to put it onto other carriers, for instance glass, thus enabling the integration of active circuitry into any conceivable material, for instance clothing and furniture. Solid-state lighting is a new technology with unprecedented possibilities in the design of novel concepts for lighting. Light sources can be made whose color and intensity can be adjusted electronically. Large area luminaries can be designed with a few millimeters thickness. The beam shape of light sources can be made electronically adjustable. Light can be integrated into cloth as is done in the photonic textiles. Advances in digital signal processing have made it possible to apply audio and video watermarks that enable conditional access, retrieval, and copy protection of audio and video material. There are many new efficient and effective standards for wireless communication. Novel communication protocols support authentication, partial information, and multiple media in a secure way. Novel media compression schemes building on MPEG4 and MPEG7 enable effective transmission and composition of video material. Recent developments in speech processing and vision technologies enable new interaction concepts that can be used in conversational user interfaces, thus allowing are a first step towards the development of natural interfaces. And this is just a list of recent technology examples. A more detailed overview of Ambient Intelligence related technology developments can be found in the book AmIware [18].

One may argue that technology is no longer the obstructive element in the development of AmI environments. Obviously, there is an abundance of technological breakthroughs that can be used to the advantage of the development of Ambient Intelligence. Examples are solid-state lighting, wearable electronics, large area display electronics, wireless sensor systems, ubiquitous communication technologies, content creation tools, 3D graphics, conversational interfaces, vision algorithms, ambient database systems, navigation tools, and many others.

One might conclude from these very developments that technology has become a commodity as it has become widely available and no longer acts as the ultimate driver of innovation. This statement however should be considered with great caution because there are still many open challenges in AmIware. For instance, wireless power delivery technologies and autonomously empowered systems are two grand research challenges from the point of view of energy management and consumption in AmI environments. Also, the distributed control of large-scale wireless 
Table 2

System Intelligence versus Social Intelligence

\begin{tabular}{ll} 
System Intelligence & Social Intelligence \\
\hline - Context aware & $\cdot$ Socialized \\
- Personalized & - Empathic \\
- Adaptive & $\cdot$ Conscious \\
- Anticipatory & \\
\hline
\end{tabular}

actuator networks is still an unresolved issue. Finally, we mention in this respect the development of intelligent always-on algorithms for the use in selforganizing learning environments is an open issue.

One of the conclusions however from the field of embedded technology is that the design and manufacturing of electronic devices has indeed reached a level of miniaturization which allows for the integration of electronic systems for processing, communication, storage display, and access into any possible physical object like clothes, furniture, cars, and homes, thus making people's environments smart.

\section{System versus social intelligence}

The AmI vision positions human needs as the key element in the development of digital innovations that enrich peoples' lives while technology is seen as a means to achieve this objective. Aspects such as information overload, violations of privacy and lack of trust in general threaten the introduction of novel technologies into our day-to-day life and consequently it is often not clear whether people will perceive such scenarios as beneficial. Essential are the user experiences people perceive when interacting with AmI environments. Examples of such experiences are immersiveness and social connectedness, which can be viewed as emergent features of intelligent behavior in AmI systems.

In the original formulation of the AmI vision intelligent systems behavior was attributed to four systems elements identified as context awareness, personalization, adaptive behavior, and anticipatory, which are defined as follows [2]; see also Table 2.

- Context aware. The environment can determine the context in which certain activities take place, where context relates meaningful information about persons and the environment, such as positioning and identification.
- Personalized. The environment can be tailored to the individual needs of users. It can recognize users and adjust its appearance to maximally support them. Automatic user profiling can capture individual user profiles through which personalized settings and information filtering can be accommodated.

-Adaptive. The environment can change in response to the users' needs. It can learn from recurring situations and changing needs, and adjust accordingly.

- Anticipatory. The environment can act upon the user's behalf without conscious mediation. It can extrapolate behavioral characteristics and generate pro-active responses.

These elements primarily facilitate intelligent communication with AmI environments thus providing users means for intelligent interaction and control. However, due to these increased expectations of AmI technologies the true intelligence of AmI environments requires complementing with social intelligence, where social means that it is compliant with societal conventions. To this end we introduce three elements of social intelligence into AmI environments identified as socialized, emphatic, and conscious. These can be formulated as follows [4].

- Socialized. User interaction concepts apply communication protocols that are compliant with societal conventions thus following social rules and commonly accepted manners and social etiquettes.

- Empathic. Interaction concepts exhibit their awareness of the inner state of emotions and motives of the user and adapt to this state accordingly by demonstrating understanding and helpful behavior.

- Conscious. The system has an inner state that exhibits a consistent and transparent behavior in its interaction with people and which is recognized by the user as conscientious. 
The two forms of intelligence evidently are interrelated in AmI environments. For example, in a sensing environment some form of system intelligence can be context aware and thus know that a person is in a private situation. A personalized system would know that it is the user's preference not to be disturbed in such situation. An intelligent system that is socialized would use common sense knowledge to not allow disturbing the person in such a context. From this simple example it should also be clear that although we make a distinction between systems and social intelligence at conceptual level they should be integrated at implementation level.

\section{New research perspectives}

Although the vision of Ambient Intelligence was introduced almost ten years ago, the development and implementation of the resulting systems and environments is still in its infancy. As with most disruptive concepts this is primarily due to the gap that exists between the fiction of the concepts resulting from the vision on the one hand and the intricacy of realization on the other hand. To discuss this issue we distinguish between the following seven topics.

1. Ambient control. How can we access and control devices in an AmI environment? The issue is how to integrate the physical world into the digital world. It concerns functionalities like sensing, actuation, tagged identification, secure wireless access, and identity management. It is concerned with collecting information about objects and their surroundings. In addition it should support for context, meaning and semantics. Finally, it should support control of distributed networks of small sensing and actuating devices that collect information from the environment and can generate physical effects through lighting devices, loud speakers, displays, ramblers, etc.

Ambient control should also support the distribution of media over physical devices so as to integrate virtual and physical worlds. It should enable the distribution of media onto physical objects and support the combination of different media including audio, video, light, smell, vibration, and airflow through into distributed media services. This calls for the extension of Quality-of-Service (QoS) into the concept of Quality of Experience (QoE). It also requires solutions to lifecycle engineering of media services in order to guarantee high quality ambient control of new media.
To provide ambient control a standardized open platform is needed. A promising approach in this respect is the Internet of Things [12], which is a novel approach to the current Internet aiming at sensing and actuation through URL's and quality controlled access layers. It extends the functionality of the current Internet which provides access to data to a new Internet architecture that supports access to physical objects. These objects may extent to the class of so-called mechatronic devices that can act on the physical world, leading to networks of pervasive sensors and actuators.

2. Tangible interfaces. What are the multi-modal interaction concepts that actors can apply in an AmI environment? The issue is to come up with new interaction concepts that extend beyond the current user interface concepts like the desktop metaphor, menu driven remote controls, and speech interfaces. Probably one of the most promising approaches in this respect is that of tangible user interfaces. This concept refers to interfaces that use physical artifacts as objects for representation and interaction. The aim is to seamlessly integrate the physical and digital worlds. The physical artifacts are often called tangible, graspable or haptic objects. The objects couple into digital information and embody the interaction. The functions in the environment that can be addressed by the objects ore called graspable functions. Moreover, they couple perceptually into the mediated action and represent key elements of the AmI environment.

Tangible user interfaces build on the co-presence of user, object, and environment. They bridge the real/physical world and virtual/digital world [21]. Input and output spaces coincide and the user is part of the interaction space. The shape, color, orientation, and size of the objects may play a role in the interaction. The objects serve as specialized input devices that support physical manipulation. In this way interaction becomes space-multiplexed. Touch control and haptic feedback may be part if the interaction concept. Separation of different tangible input devices over different functions may lead to distributed and/or simultaneous interaction with Ami environments.

The interaction resulting from tangible user interfaces is not mediated and it supports direct engagement of the user with the environment. Consequently it is definitely more intuitive and therefore more natural than the currently known user interfaces. However, the coupling between the tangibles and mean- 
ingful graspable functions is to a large extent not well-understood [14].

3. End-user Programming. How can end-users program their own functionalities into an AmI environment? The issue is to design programming environments that allow non-computer experts to create and modify software artifacts in an AmI environment. It should empower end-users to tailor the environments towards their needs. It involves methods and tools for editing, interpreting, linking, executing, and rendering of application functions in are user friendly and intuitive way [16].

It is generally believed that successful approaches to end-user programming should use metaphors, pictograms, and icons within an application-specific setting to specify actions at intentional level. Wellknown notions to indicate the various approaches are visual programming, graphical programming, programming by example, and programming by rehearsal [15]. End-user programming environments should show the immediate feedback of the programming actions either through $3 \mathrm{D}$ visualization, animation, or simulation. Furthermore, it should be possible to render the effect of the action onto the AmI environment through the available Ambient Control system. Furthermore, the end-user programming environment should support the creation and modification of the natural interface concepts defined for the AmI environment.

End-user programming starts with scenario building. These scenarios describe sequences of nonmonotonic actions that can occur in an AmI environment. Such scripts are often referred to as ambient narratives [10]. They relate to high level descriptions of features or experiences that emerge from situated actions a participative actor (user) takes in an AmI environment. The translation of these actions into system properties involves 3D modeling, interaction scripting, process-modeling, non-monotonic reasoning over temporal constraints, and device rendering. As a final element we mention the need for life-cycle management of ambient narratives.

4. Sensory Experiences. How de people process information in the interaction with AmI environments? The issue is to understand the interaction between the human brain and its environment. This field is concerned with the investigation of fundamental and essential functions of the brain, including perception, thinking, emotion, learning, memory, attention, heuristic search, planning, reasoning, discovery, and creativity [8]. It involves elements like modeling of human brain information processing; simulation of learning and reasoning mechanisms, modeling of human memory storage and recollection processes. Understanding emotions is essential to the creation and/or recreation of experiences [25]. Key questions are how to measure the emotional state of users in a reliable way and how to use this knowledge to enhance the interaction with AmI environments. Capturing, influencing, and generating emotions is a new field of research, that is often referred to as affective computing. Key research question are: how can we model a mood state, which moods do we need to be able to capture, and how does triggering a set of senses influence the perceived emotions?

Sensing and actuation of sensorial effects in relation to human activity are important elements in the development of effective solutions in sensory experiences. The entire field of human sensing and actuation is devoted to this subject and in a way one can state that this field is still in its infancy. It is concerned with the investigation of all kinds of physical parameters and effects and that can influence human emotions. Key research questions are: how can we derive descriptors for user mood/emotions based on emotion-capturing input? How can we generate or render appropriate emotional effects. Many of the answers to these questions can be found in the domain of perception research, which is a research field at the intersection of cognitive psychology and electrical engineering. The next big issue is how to incorporate peoples' mood in the interplay with content to result at positive experiences? For this we need a scientific framework to capture, measure, quantify, judge, and explain the user experience.

5. Social presence. How can mediated social interaction be achieved in AmI environments? This is about being there and being together. The issue is to convey a certain degree of salience of persons interacting through AmI environments or in other words how can AmI environments be support social aspects interpersonal interaction? Biocca et al., [9] distinguish the following three basic elements in social presence.

- Co-presence; the degree to which another person or its avatar is perceived as "real" in the mediated communication or in other words the sensory mutual awareness of the embodied other.

- Psychological involvement; the degree of salience of another person in the interaction and the resulting salience of the interpersonal relationship or in 
other words the access to the intelligence of the other person in the interaction.

- Behavioral engagement; the degree to which behavior is synchronized in the interraction between mediated actors or in other words the behavior expressed during the engagement of a person with the AmI environment.

Important research questions relate to topics such as the perceived social richness of the medium, the involvement, immediacy, and intimacy of the interaction, and the social judgment of the interacting persons. The entire field of social presence is not well understood to a large extent because of the lack of a genuine theory from which measures can be derived. An appropriate theory of social presence should address the following main topics.

- It should specify the range of phenomena we seek to understand,

- delimit the range of causal relationships of the phenomena that need to be explained,

- determine predicted behavior and its range, and

- provide a guidance for the design of social behavior of AmI environments.

6. Trustful persuasion. How can AmI environments support context sensitive system behavior and deliver persuasive content and interaction in a trustful manor? According to Fogg [11] we can identify the following issues related to persuasion.

- Reduction replaces a complex task by a simpler one by virtue of the introduction of automation and computation, but also by virtue of anticipation and defining characteristics of Ambient Intelligence.

- Customization and tailoring adjusts messages and content to the beliefs and needs of the person. This requires very rich, privacy sensitive models of users that go beyond simple habits and preferences, and include aspects of their personality, their health status, the social network and context, etc

- Suggestion reminds people to perform certain behaviors at opportune moments. Prompting of behaviors then needs to be sensitive to context, which is a central aspect of Ambient Intelligence.

- Self-monitoring allows people to monitor themselves and to inform them about how they could modify their behaviors. Self-monitoring can be very tedious; it will be argued below that Ambi- ent Intelligence opens up the opportunity to facilitate this process and thus achieve persuasion.

A particularly promising domain for studying persuasion concerns health care and more specifically the question how people will be motivated to adopt healthier life-styles. In the domain of medicine the general problem of persuasion has been called compliance, which is defined as the extent to which a person's behavior coincides with medical or health advice [26]. Compliance is recognized as a major challenge in medical research, especially when treatment protocols are individualized or situationdependent or where the patient is unsupervised and so reporting on compliance is not reliable. The challenge is to develop new approaches that can monitor compliance behavior and can trigger persuasive interventions.

7. e-Inclusion and ethics. What does it take for people to accept that their environment is monitoring their every move, waiting for the right moment to take over for the purpose of taking care of them? Much of this acceptance will depend on the perceived functional benefit of AmI environments and on the availability of mechanisms that enable participants to make their own choices in a way that is understandable, transparent, and independent of their comprehension level. These mechanisms should comply with the basic agreements that have been formulated in the European Charter of Fundamental Rights, which are concerned with properties such as autonomy, freedom of choice and self-determination. The solution to this problem is often communicated as embedded ethics which try to handle the fundamental rights already at the level of the design of the corresponding embedded systems.

Of a different scale are the concerns that are raised by the fact that personalization requires registration and recording of user behavior [13]. The explicit knowledge about a so-called digital soul of human beings requires the development of different standards for social behavior, and it might even be desired to protect people against their own attitude. Finally, people raise their concerns against the absolutistic technological nature of Ambient Intelligence. Pushing Ambient Intelligence to the extreme might lead to a world full of digital surrogates for about everything that is conceivable. An in-depth study of dark scenarios for Ambient Intelligence was conducted by the SWAMI (Safeguards in a World of Ambient Intelligence) group, who published a long list of concerns that might be raised by the introduc- 
Table 3

Positioning Experience Research

\begin{tabular}{lll} 
Experience@Context & Experience@Lab & Experience@Field \\
\hline - Trend studies & • Concept definition & - Field tests \\
• Insight generation & • Experience prototyping & - Longitudinal studies \\
- Insight validation & • User centered design & - Trials \\
& and engineering & \\
\hline
\end{tabular}

$\begin{array}{lll}\text { Society } & \text { Laboratory } & \text { Real-Life }\end{array}$

tion of AmI concepts [22]. This seminal study provides an excellent starting point for the discussion of the ethics of Ambient Intelligence as it approaches the issue in a well-structured and systematic way covering any conceivable angle.

Another ethical concern is given by possibility to incorporate Ambient Intelligence into an even more intimate ambience - into our own bodies. We are already incorporating intelligence into our clothing, and we are quite happy to have a pacemaker built into our bodies. Targeted drug delivery or minimally invasive surgery approaches are medical treatments based on electronics embedded into our bodies. Evidently, this has a medical justification. But how long will it be before we accept the implantation of chips for non-medical reasons? Attitudes to the body are already changing. Body piercing, tattoos and cosmetic surgery are much more common than a generation ago. More recently, the company Applied Digital Solutions received the go-ahead from the FDA to market a chip that can be injected into children or Alzheimer's patients, so that they can be traced by GPS. If this sort of product finds wide-spread public acceptance, will we have crossed an important boundary? Where will people draw the line between the organic and the inorganic, the real and the artificial? And how will that affect how we view and treat our AmI environments, and each other.

\section{Experience research}

The development of novel AmI environments requires new ways of working as the ultimate goal is to position the user in the center of the interaction. This implies that the user should play an active role in the research process. This can be done by introducing the concept of Experience Research, which is aimed at developing methods and techniques that allow the validated feedback of users in the process of generating experiences [1]. To account for this we distinguish between the following three elements in relation to experience research; see also Table 3.

Experience@Context is aimed at elicitating and validating End-user insights from target groups in their societal context. These insights should reveal unmet needs which can provide a starting point for the development of new solutions. Therefore, we need a deeper understanding of the nature of human behavior, not from a marketing point emphasizing consumer behavior, but from a deeper personality point of view emphasizing basic human needs and emotions. In the elicitation of these insights no solutions should be taken into account and they should be validated in such a way that they are statistically significant. Furthermore, the insights should contain the two following two elements. First they should be allow for deep customization of AmI environments by casting the behavioral insights into computational models that capture the human emotions and provide the AmI environments with the social intelligence as defined above. Second, the end-user insights should provide clear anchor points for novel concept market combinations of solutions that really make sense in the eyes of users.

Experience@Laboratory uses controlled laboratory environments to support the execution of usercentered design cycles in the development of novel interaction concepts and solutions. AmI environments can no longer be considered as closed-box devices that can be designed and developed on the bases of a number of use-cases that are translated into well-defined system requirements. AmI environments are highly interactive and responsive and their emergent behavior can only be implicitly defined and specified. Consequently, such systems can only be 
designed through processes that allow for iterative engineering and co-creation. This calls for research facilities that support fast and effective development and realization of novel concepts through experience prototypes that can be refined through in sequence of design and engineering cycles in which additional information is obtained and effectuated through user evaluations. These facilities should be as realistic as possible so as to allow users to interact with the experience prototypes is a natural setting. However, they should be highly controllable in order maintain test situations stable and eliminate at much as possible unwanted artifacts.

Experience@Field refers to studies in which novel concepts and solutions are tested in real-life settings which are not controlled. As a result the tests are carried out in the actual situation in which the concepts and solutions will be used eventually. This typically calls for longitudinal field tests and studies in which users are exposed to certain propositions in normal daily-life situations. The resulting feedback is of utmost relevance since it provides information about the natural setting in which the proposed concepts and solutions are going to be used. The Experience@Field approach can capture aspects that are not covered by the other elements in experience research as it applies to an actual real-life situation in which all normal real-life factors and their interrelation play a role, which often cannot be accounted for in the controlled laboratory setting. Examples of such factors are stress and emotions, which have a strong influence on the perceived interaction of individuals with their AmI environments.

\section{Conclusion}

It goes without saying that the AmI vision holds the promise of becoming a truly disruptive paradigm. It calls for a far-reaching multi-disciplinary and integrative approach that extends far beyond the levels of system innovation that mankind has been dealing with so far. This requirement is a challenge and a threat at the same time. The threat lies in the fact that the complexity of AmI environments may not be tractable and that the implementation of the vision therefore will be infeasible. On the other hand the requirement may stimulate the search for innovative solutions to this complexity problem resulting into now insights that eventually will lead to the realization of true Ambient Intelligence.

\section{References}

[1] E.H.L. Aarts and E. Diederiks, Ambient Lifestyle: From Concept to Experience, BIS Publishers Amsterdam, 2007.

[2] E.H.L. Aarts, H. Harwig, and M. Schuurmans (2001), Ambient Intelligence, in: J. Denning (ed.) The Invisible Future, McGraw Hill, New York, NY, USA, pp. 235-250.

[3] E.H.L. Aarts and J. Encarnaçao (2006), True Visions: The Emergence of Ambient Intelligence, Springer, Berlin, Germany.

[4] E.H.L. Aarts, P. Markopoulos, and B. de Ruyter (2007), The persuasiveness of Ambient Intelligence, in: M. Petkovic and W. Jonker (eds.), Privacy and Trust in Modern Data Management, Springer, Berlin, Germany, pp. 367-381.

[5] E.H.L. Aarts and S. Marzano (eds.) (2003), The New Everyday: Visions of Ambient Intelligence, 010 Publishing, Rotterdam, The Netherlands.

[6] F. Adelstein, S.K.S. Gupta, G.G. Richard, and L. Schwiebert (2004), Fundamentals of Mobile Computing and Pervasive Computing, McGraw Hill, New York, NJ, USA.

[7] J.C. Augusto and D. Shapiro (eds.) (2007), Advances in Ambient Intelligence, Frontiers in Artificial Intelligence and Applications, Volume 164, IOS Press, Amsterdam, The Netherlands.

[8] R. Backs and W. Boucsein (eds.) (2000), Engineering Psychophysiology: Issues and Applications, Lawrence Erlbaum Associates, New York, NJ, USA

[9] F. Biocca, C. Harms, and J.K. Burgoon (2003), Toward a more robust theory and measure of social presence; review and suggested criteria, Presence 12(5), 456-480.

[10] M. Doorn and A.P. de Vries (2006), Co-creation in ambient narratives, in: Y. Cai and J. Abascal (eds.), Ambient Intelligence in Everyday Lives, Springer, Berlin, Germany, pp. 103-129.

[11] B.J. Fogg (2002), Persuasive Technology, Morgan Kaufmann, San Francisco, CA, USA.

[12] N. Gershenfeld, R. Krikorian, and D. Cohen (2004), The Internet of Things, Scientific American 291(4), 76-81.

[13] M. Hildebrandt and S. Gutwirth (eds.) (2008), Profiling the European Citizen, Springer, Berlin, Germany.

[14] E. Hornecker and J. Buur (2006), Getting a grip on tangible interaction: A frame work on physical space and social interaction, Proceedings of the International Conference on Computer Human Interaction, Montreal, Canada, ACM Press, pp. 437-446.

[15] C. Kelleher and R. Pausch (2005), Lowering the barriers to programming: A taxonomy of programming environments and languages for novice programmers, ACM Computing Surveys 37(2), 83-137.

[16] H. Lieberman (ed.) (2001), Your Wish is my Command, Morgan Kaufmann, San Francisco, CA, USA.

[17] R. Noyce (1977), Microelectronics, Scientific American 237(3), 63-69.

[18] S. Muhkerjee, E.H.L. Aarts, M. Ouwerkerk, R. Rovers, and F. Widdershoven (eds.) (2005), AmIware: Hardware Drivers for Ambient Intelligence, Springer, Berlin, Germany.

[19] R. Picard (1997), Affective Computing, The MIT Press, Cambridge, MA, USA.

[20] M. Satyanarayanan (2001), Pervasive Computing: Vision and Challenges, IEEE Personal Communications 8(4), 10-17.

[21] B. Ullmer and H. Ishii (2001), Emerging frameworks for tangible user interfaces, in: J.M. Carroll (ed.), Human Computer Interaction in the New Millennium, Addison Wesley, Reading, MA, USA, pp. 579-601. 
[22] D. Wright, S. Gutwirth, M. Friedewald, E. Vildjiounaite, and Y. Punie (eds.) (2008), Safeguards in a World of Ambient Intelligence, Springer, Berlin, Germany.

[23] W. Weber, J. Rabaey, and E. Aarts (eds.) (2005), Ambient Intelligence, Springer Verlag, Berlin, Germany.

[24] M. Weiser (1991), The Computer for the Twenty-First Century, Scientific American 165(3), 94-104.
[25] J. Westerink, M. Ouwerkerk, T.J.M. Overbeek, F. Pasveer, and B. de Ruyter, Probing Experiences, Springer, Berlin, Germany.

[26] S. Winnick, D.O. Lucas, A. Hartman, and D. Toll (2005) How do you improve Compliance? Pediatrics 115(6), 718 724. 